\title{
Quantum Density Fluctuations in Classical Liquids
}

\author{
L.H. Ford* \\ Institute of Cosmology \\ Department of Physics and Astronomy \\ Tufts University, Medford, MA 02155 \\ N.F. Svaiten $\dagger$ \\ Centro Brasiliero de Pesquisas Fisicas CBPF \\ Rua Dr. Xavier Sigaud 150 \\ Rio de Janeiro, RJ, 22290 180, Brazil
}

\begin{abstract}
We discuss the density fluctuations of a fluid due to zero point motion. These can be regarded as density fluctuations in the phonon vacuum state. We assume a linear dispersion relation with a fixed speed of sound and calculate the density correlation function. We note that this function has the same form as the correlation function for the time derivative of a relativistic massless scalar field, but with the speed of light replaced by the speed of sound. As a result, the study of density fluctuations in a fluid can be a useful analog model for better understanding fluctuations in relativistic quantum field theory. We next calculate the differential cross section for light scattering by the zero point density fluctuations, and find a result proportional to the fifth power of the light frequency. This can be understood as the product of fourth power dependence of the usual Rayleigh cross section with the linear frequency dependence of the spectrum of zero point density fluctuations. We give some estimates of the relative magnitude of this effect compared to the scattering by thermal density fluctuations, and find that it can be of order $0.5 \%$ for water at room temperature and optical frequencies. This relative magnitude is proportional to frequency and inversely proportional to temperature. Although the scattering by zero point density fluctuation is small, it may be observable.
\end{abstract}

PACS numbers: 05.40.-a,46.65.+g,62.60.+v,78.35.+c 
Zero point motion is a well established phenomenon, both in condensed matter physics and relativistic quantum field theory. One effect of the zero point motion of atoms is that the Debye-Waller factor does not approach unity at zero temperature, but is typically about 0.9 at low temperature [1]. This means that the intensity of X-ray diffraction lines is reduced by approximately $10 \%$ as a result of atomic zero point motion. In quantum field theory, an example of a zero point phenomenon is the Casimir effect [2], the force of attraction of bodies due to shifts in electromagnetic zero point energy. There is an analog of the Casimir effect in which zero point fluctuations of the phonon field in a fluid also produce an analogous force [3]. Unfortunately, the phononic analog is smaller than the electromagnetic effect by the ratio of the speed of sound to the speed of light, and is hence very small. Some authors have discussed the possibility of an "acoustic Casimir" effect produced not by zero point fluctuations, but rather by a thermal or stochastic bath of sound [4, 5]. Other authors have recently discussed acoustic analogs of the Casimir effect in thin films [6] and in Bose-Einstein condensates [7] or other quantum liquids [8]. It has recently been argued that the thermal effects in liquid helium may be large enough to observe [9]. In the present paper, we will be primarily concerned with local density fluctuations in the phonon vacuum state. We also deal with classical as opposed to quantum liquids, so the the deBroglie wavelength of the atoms is small compared to the interatomic separation.

Here we consider the quantization of sound waves in a fluid with a linear dispersion relation, $\Omega_{q}=c_{S} q$, where $\Omega_{q}$ is the phonon angular frequency, $q$ is the magnitude of the wave vector, and $c_{S}$ is the speed of sound in the fluid. This should be a good approximation for wavelengths much longer the interatomic separation. Let $\rho_{0}$ be the mean mass density of the fluid. Then the variation in density around this mean value is represented by a quantum operator, $\hat{\rho}(\mathbf{x}, t)$, which may be expanded in terms of phonon annihilation and creation operators as [10]

$$
\hat{\rho}(\mathbf{x}, t)=\sum_{\mathbf{q}}\left(b_{\mathbf{q}} f_{\mathbf{q}}+b_{\mathbf{q}}^{\dagger} f_{\mathbf{q}}^{*}\right)
$$

where

$$
f_{\mathbf{q}}=\sqrt{\frac{\hbar \omega \rho_{0}}{2 V c_{S}^{2}}} \mathrm{e}^{i\left(\mathbf{q} \cdot \mathbf{x}-\Omega_{q} t\right)} .
$$

Here $V$ is a quantization volume. The normalization factor in Eq. (2) can be fixed by requiring that the zero point energy of each mode be $\frac{1}{2} \hbar \Omega_{q}$ and using the expression for the energy density in a sound wave,

$$
U=\frac{c_{S}^{2}}{\rho_{0}} \hat{\rho}^{2}
$$

In the limit in which $V \rightarrow \infty$, we may write the density correlation function as

$$
\left\langle\hat{\rho}(\mathbf{x}, t) \hat{\rho}\left(\mathbf{x}^{\prime}, t^{\prime}\right)\right\rangle=\frac{\hbar \rho_{0}}{16 \pi^{3} c_{S}^{2}} \int d^{3} q \Omega_{q} \mathrm{e}^{i\left(\mathbf{q} \cdot \Delta \mathbf{x}-\Omega_{q} \Delta t\right)},
$$

where $\Delta \mathbf{x}=\mathbf{x}-\mathbf{x}^{\prime}$ and $\Delta t=t-t^{\prime}$. The integral may be evaluated to write the coordinate space correlation function as

$$
\left\langle\hat{\rho}(\mathbf{x}, t) \hat{\rho}\left(\mathbf{x}^{\prime}, t^{\prime}\right)\right\rangle=-\frac{\hbar \rho_{0}}{2 \pi^{2} c_{S}} \frac{\Delta \mathbf{x}^{2}+3 c_{S}^{2} \Delta t^{2}}{\left(\Delta \mathbf{x}^{2}-3 c_{S}^{2} \Delta t^{2}\right)^{3}} .
$$

This is of the same form as the correlation function for the time derivative of a massless scalar field in relativistic quantum field theory, $\left\langle\dot{\varphi}(\mathbf{x}, t) \dot{\varphi}\left(\mathbf{x}^{\prime}, t^{\prime}\right)\right\rangle$. Apart from a factor of $\rho_{0}$, 
these two quantities may be obtained from one another by interchanging the speed of light $c$ and the speed of sound $c_{S}$. If $c \rightarrow c_{S}$, then

$$
\left\langle\dot{\varphi}(\mathbf{x}, t) \dot{\varphi}\left(\mathbf{x}^{\prime}, t^{\prime}\right)\right\rangle \rightarrow \rho_{0}\left\langle\hat{\rho}(\mathbf{x}, t) \hat{\rho}\left(\mathbf{x}^{\prime}, t^{\prime}\right)\right\rangle .
$$

In the limit of equal times, the density correlation function becomes

$$
\left\langle\hat{\rho}(\mathbf{x}, t) \hat{\rho}\left(\mathbf{x}^{\prime}, t\right)\right\rangle=-\frac{\hbar \rho_{0}}{2 \pi^{2} c_{S}(\Delta \mathbf{x})^{4}} .
$$

Thus the density fluctuations increase as $|\Delta \mathbf{x}|$ decreases. Of course, the continuum description of the fluid and the linear dispersion relation both fail as $|\Delta \mathbf{x}|$ approaches the interatomic separation. Also note the minus sign in Eq. (17). This implies that density fluctuations at different locations at equal times are anticorrelated. By contrast, when $c_{S}|\Delta t|>|\Delta \mathbf{x}|$, then $\left\langle\hat{\rho}(\mathbf{x}, t) \hat{\rho}\left(\mathbf{x}^{\prime}, t\right)\right\rangle>0$ and the fluctuations are positively correlated. This is complete analogy with the situation in the relativistic theory. Fluctuations inside the lightcone can propagate causally and tend to be positively correlated. Fluctuations in a fluid for which $c_{S}|\Delta t|<|\Delta \mathbf{x}|$ cannot have propagated from one point to the other, and are anti-correlated. This can be understood physically because an over density of fluid at one point in space requires an under density at a nearby point.

Thus the quantum density fluctuations in a fluid can serve as an analog model for fluctuations in quantum field theory. The effect of boundaries on the density fluctuations is similar to the effect of reflecting boundaries on the mean squared electric and magnetic fields. The effects of various types of boundaries on the density fluctuations will be treated in detail in another paper [11], but as an example we here quote the result for a planar boundary. At an impenetrable boundary, the normal derivative of the fluid density must vanish. This is corresponds to a massless scalar field which satisfies Neumann boundary conditions. We can define the shift in the mean squared density, $\left\langle(\delta \rho)^{2}\right\rangle_{R}$ as

$$
\left\langle(\delta \rho)^{2}\right\rangle_{R}=\lim _{\Delta t \rightarrow 0, \Delta \mathbf{x} \rightarrow 0}\left[\left\langle\hat{\rho}(\mathbf{x}, t) \hat{\rho}\left(\mathbf{x}^{\prime}, t^{\prime}\right)\right\rangle_{B}-\left\langle\hat{\rho}(\mathbf{x}, t) \hat{\rho}\left(\mathbf{x}^{\prime}, t^{\prime}\right)\right\rangle\right]
$$

where \langle\rangle$_{B}$ denotes the correlation function in the presence of a boundary. At a distance $z$ from a planar boundary, we find

$$
\left\langle(\delta \rho)^{2}\right\rangle_{R}=-\frac{\hbar \rho_{0}}{32 \pi^{2} c_{S} z^{4}} .
$$

Note the minus sign, which indicates a reduction in fluctuations near the boundary. This is analogous to the shift in the mean squared electric and magnetic fields near a perfectly reflecting plate [12] (Electromagnetic quantities will in Lorentz-Heaviside units throughout this paper.)

$$
\left\langle E^{2}\right\rangle=-\left\langle B^{2}\right\rangle=\frac{3 \hbar c}{16 \pi^{2} z^{4}}
$$

Next we turn to the question of whether these zero point density fluctuations are observable. Two means for detecting density fluctuations are by light scattering or by neutron scattering. We consider only the former here. The scattering of light by thermal fluctuations has been extensively studied [13] in the past. One approach utilizes the Maxwell equations with a fluctuating dielectric function. Write the dielectric function of the fluid as $\epsilon=\epsilon_{0}+\epsilon_{1}$, 
with $\epsilon_{0}$ being the mean dielectric constant of the fluid, and $\epsilon_{1}$ the fluctuating part. We assume that the dielectric function is proportional to density, so we can write

$$
\epsilon_{1}(\mathbf{x}, t)=\frac{\epsilon_{0}}{\rho_{0}} \hat{\rho}(\mathbf{x}, t)
$$

One may then use results such as Eq. (1.68) of Ref. [13] to obtain the scattering cross section. However, here we will summarize a different approach which leads to the same result.

Consider the quantized electromagnetic field in a non-dispersive dielectric with dielectric constant $\epsilon_{0}$. The Hamiltonian may be written in terms of the electric and magnetic fields as

$$
H_{0}=\frac{1}{2} \int d^{3} x\left(\epsilon_{0} \mathbf{E}^{2}+\mathbf{B}^{2}\right)
$$

Here the electric field operator may be expanded in photon annihilation and creation operators as

$$
\mathbf{E}(\mathbf{x}, t)=\sum_{\mathbf{k}, \lambda} \sqrt{\frac{\hbar \omega}{2 V \epsilon_{0}}}\left[a_{\mathbf{k}, \lambda} \hat{\mathbf{e}}_{\mathbf{k}, \lambda} \mathrm{e}^{i(\mathbf{k} \cdot \mathbf{x}-\omega t)}+a_{\mathbf{k}, \lambda}^{\dagger} \hat{\mathbf{e}}_{\mathbf{k}, \lambda} \mathrm{e}^{-i(\mathbf{k} \cdot \mathbf{x}-\omega t)}\right],
$$

where $\hat{\mathbf{e}}_{\mathbf{k}, \lambda}$ are real polarization vectors and $\lambda$ labels linear polarization states. Here

$$
\omega=\frac{c}{\sqrt{\epsilon_{0}}} k
$$

Suppose that the electromagnetic field is coupled to the dielectric fluctuations by the interaction Hamiltonian

$$
H^{\prime}=\frac{1}{2} \int d^{3} x \epsilon_{1}(\mathbf{x}, t) \mathbf{E}^{2}(\mathbf{x}, t)
$$

We wish to calculate the amplitude for a photon in an initial state $(\mathbf{k}, \lambda)$ to scatter into state $\left(\mathbf{k}^{\prime}, \lambda^{\prime}\right)$ with the emission of a phonon into mode $\mathbf{q}$. Thus the initial state of the photon + phonon system is $\left|\psi_{i}\right\rangle=\left|1_{\mathbf{k}, \lambda}, 0_{\mathbf{q}}\right\rangle$, and the final state is $\left|\psi_{f}\right\rangle=\left|1_{\mathbf{k}^{\prime}, \lambda^{\prime}}, 1_{\mathbf{q}}\right\rangle$. We use first order perturbation theory and write

$$
\left\langle\psi_{f}\left|H^{\prime}\right| \psi_{i}\right\rangle=\sqrt{\frac{\hbar^{3} \omega \omega^{\prime} \Omega_{q}}{8 V \rho_{0} c_{S}^{2}}}\left(\hat{\mathbf{e}}_{\mathbf{k}, \lambda} \cdot \hat{\mathbf{e}}_{\mathbf{k}^{\prime}, \lambda^{\prime}}\right) \delta_{\mathbf{k}, \mathbf{k}^{\prime}+\mathbf{q}} \mathrm{e}^{i\left(\omega^{\prime}+\Omega_{q}-\omega\right) t} .
$$

The transition rate is given by the usual relation

$$
\mathcal{W}=\frac{2 \pi}{\hbar}\left|\left\langle\psi_{f}\left|H^{\prime}\right| \psi_{i}\right\rangle\right| \rho_{f}
$$

where the density of photon final states in energy is here given by

$$
\rho_{f}=\frac{V\left(\omega^{\prime}\right)^{2} \epsilon_{0}^{\frac{3}{2}}}{\hbar(2 \pi c)^{3}} d \Omega
$$

for scattering into solid angle $d \Omega$. The incident flux of photons is given by $c /\left(V \sqrt{\epsilon_{0}}\right)$. This leads to the result for the photon scattering cross section by zero point fluctuations

$$
\left(\frac{d \sigma}{d \Omega}\right)_{Z P}=\frac{\hbar \omega\left(\omega^{\prime}\right)^{3} \Omega_{q} \mathcal{V} \eta^{4}}{32 \pi^{2} c^{4} c_{S}^{2} \rho_{0}}\left(\hat{\mathbf{e}}_{\mathbf{k}, \lambda} \cdot \hat{\mathbf{e}}_{\mathbf{k}^{\prime}, \lambda^{\prime}}\right)^{2},
$$


where $\mathcal{V}$ is the scattering volume and $\eta \sqrt{\epsilon_{0}}$ is the fluid's index of refraction. This relation may also be derived from classical electromagnetic theory with a fluctuating dielectric.

The conservation of energy and momentum require that

$$
\omega=\omega^{\prime}+\Omega_{q}
$$

and that

$$
\mathbf{k}=\mathbf{k}^{\prime}+\mathbf{q}
$$

The frequency of the created phonon, $\Omega_{q}$, is small compared to the light frequency $\omega$, so that $\omega^{\prime} \approx \omega$ and one may show that

$$
\Omega_{q} \approx \sqrt{2(1-\cos \theta)} \frac{c_{S}}{c} \omega
$$

where $\theta$ is the scattering angle. We can now write the cross section as

$$
\left(\frac{d \sigma}{d \Omega}\right)_{Z P}=\sqrt{2(1-\cos \theta)} \frac{\hbar \omega^{5} \mathcal{V} \eta^{4}}{32 \pi^{2} c^{5} c_{S} \rho_{0}}\left(\hat{\mathbf{e}}_{\mathbf{k}, \lambda} \cdot \hat{\mathbf{e}}_{\mathbf{k}^{\prime}, \lambda^{\prime}}\right)^{2}
$$

The $\omega^{5}$ dependence of the scattering cross section can be viewed as the product of the $\omega^{4}$ dependence of Rayleigh-Brillouin scattering and one power of $\Omega_{q}$, and hence of $\omega$, coming from the spectrum of zero point fluctuations in the fluid. Because light travels through the fluid at speeds much greater than the sound speed, light scattering reveals a nearly static distribution of density fluctuations. Thus we can regard Eq. (23) as a probe of the fluctuations described by Eq. (7).

The scattering by zero point fluctuations is inelastic, with the creation of a phonon. Thus, the scattering described by Eq. (23) is really Brillouin rather than Rayleigh scattering. However, the result is similar to that for Brillouin and Rayleigh scattering by thermal density fluctuation is a fluid, for which the cross section at temperature $T$ is [See, for example Eq. (8.3) of Ref. [13].]

$$
\left(\frac{d \sigma}{d \Omega}\right)_{T}=\frac{\omega^{4} \mathcal{V} k_{B} T}{16 \pi^{2} c^{4}}\left[\beta_{S} \rho_{0}^{2}\left(\frac{\partial \epsilon}{\partial \rho_{0}}\right)_{S}^{2}+\frac{T}{\rho_{0} C_{P}}\left(\frac{\partial \epsilon}{\partial T}\right)_{P}^{2}\right]\left(\hat{\mathbf{e}}_{\mathbf{k}, \lambda} \cdot \hat{\mathbf{e}}_{\mathbf{k}^{\prime}, \lambda^{\prime}}\right)^{2} .
$$

Here $k_{B}$ is Boltzmann's constant, $\beta_{S}$ is adiabatic compressibility, and $C_{P}$ is the heat capacity per unit mass at constant pressure. The subscripts $S$ and $P$ refer to derivatives of the dielectric function at constant entropy and constant pressure, respectively. The two terms on the right hand side of Eq. (24) have distinct physical interpretations. The first gives the cross section for Brillouin scattering, inelastic scattering involving either the emission or absorption of a phonon, leading to the Stokes and anti-Stokes lines, respectively. The second term gives the cross section for Rayleigh scattering. Because the scattering by zero point density fluctuations involves the emission of a phonon, it will contribute to the Stokes line. Thus, we should compare Eq. (23) with the Brillouin scattering cross section

$$
\left(\frac{d \sigma}{d \Omega}\right)_{T B}=\frac{\omega^{4} \mathcal{V} k_{B} T}{16 \pi^{2} c^{4} c_{S}^{2} \rho_{0}}\left[\rho_{0}\left(\frac{\partial \epsilon}{\partial \rho_{0}}\right)_{S}\right]^{2}\left(\hat{\mathbf{e}}_{\mathbf{k}, \lambda} \cdot \hat{\mathbf{e}}_{\mathbf{k}^{\prime}, \lambda^{\prime}}\right)^{2}
$$

where we have used the relation

$$
\beta_{S}=\frac{1}{\rho_{0} c_{S}^{2}}
$$


The ratio of the zero point and thermal Brillouin cross sections can be written as

$$
R \equiv \frac{(d \sigma / d \Omega)_{Z P}}{(d \sigma / d \Omega)_{T B}}=\sqrt{2(1-\cos \theta)}\left(\frac{\hbar \omega}{2 k_{B} T}\right)\left(\frac{c_{S}}{c}\right) \eta^{4}\left[\rho_{0}\left(\frac{\partial \epsilon}{\partial \rho_{0}}\right)_{S}\right]^{-2} .
$$

The index of refraction, $\eta$, and the quantity $\rho_{0}\left(\partial \epsilon / \partial \rho_{0}\right)_{S}$ are both of order unity, so $R$ is primarily determined by the ratio of the photon energy to the thermal energy, and the ratio of the speed of sound to the speed of light.

As an example, consider the case of water at room temperature and violet light with a wavelength of $\lambda=350 \mathrm{~nm}$. In this case, we have $c_{S}=1480 \mathrm{~m} / \mathrm{s}$ and $\eta=1.4$ [14]. In addition, $\rho_{0}\left(\partial \epsilon / \partial \rho_{0}\right)_{S}=0.79$ [15]. For back scattering, $\cos \theta=-1$, this leads to $R \approx 0.005$. Consequently, about $0.5 \%$ of the Stokes line is due to zero point motion effects. Although this is a small fraction, it may be detectable, and will increase at lower temperatures and shorter wavelengths.

In summary, we have argued that the zero point density fluctuations in a fluid are of interest both as an analog model for fluctuations in relativistic quantum field theory, and in their own right. These fluctuations are potentially observable in light scattering experiments.

This work was supported in part by the National Science Foundation under Grant PHY0555754 and by Conselho Nacional de Desenvolvimento Cientifico e Tecnologico do Brasil (CNPq).

* Email: ford@cosmos.phy.tufts.edu

$\dagger$ Email: nfuxsvai@cbpf.br

[1] See, for example, C. Kittel, Introduction to Solid State Physics, 8th ed (Wiley, Hoboken, NJ, 2005), Appendix A.

[2] H.B.G. Casimir, Proc. K. Ned. Akad. Wet. B51, 793 (1948).

[3] I.E. Dzyaloshinskii, E.M. Lifshitz and L.P. Pitaevski, Adv. Phys. 10, 165 (1961).

[4] A. Larraza, Phys. Lett. A 248, 151 (1998).

[5] O. Bschorr, J. Acoust. Soc. Am. 106, 3730 (1999).

[6] E. Schäffer and U. Steiner, Eur. Phys. J. E 8, 347 (2002).

[7] D.C. Roberts and Pomeau, Phys. Rev. Lett. 95, 145303 (2005).

[8] A. Recati, J.N. Fuchs, C.S. Peça, and W. Zwerger, Phys. Rev. A 72, 023616 (2005).

[9] S.K. Lamoreaux, arXiv:0808.4000.

[10] See, for example, E.M. Lifshitz and L.P. Pitaevski, Statistical Physics, Part 2, 2nd ed. (Pergomon, Oxford, 1969), Eq. (24.10).

[11] L.H. Ford and N.F. Svaiter, manuscript in preparation. 
[12] See, for example, V. Sopova and L.H. Ford, Phys. Rev. D 66, 045026 (2002).

[13] W. Hayes and R. Loudon, Scattering of Light by Crystals, (Wiley, New York, 1978).

[14] CRC Handbook of Chemistry and Physics, D,R. Lide, ed., 89th edition (CRC Press, Boca Raton, 2008), pp 14-44 and 10-251.

[15] H.Z. Cummins and R.W. Gammon, J. Chem. Phys. 44, 2785 (1966), Table III. 